\title{
The development of tourism management system design
}

\author{
Dongying Zheng \\ Weifang University of Science and Technology, ShanDong ShouGuang, 262700, China; \\ E-mail:zdy7817@163.com
}

\begin{abstract}
Keywords:Tourism management; The JSP. Mysis; User Management; Information Query
\end{abstract}
\begin{abstract}
The design and development of tourism information management system provide a platform operation, can divide the tourism information module and classified management. The article main content includes: the requirements analysis of the system; the concept of database structure design; The database logical structure design; overall design of the database which mainly introduced the design and management module information query module process design. Finally presented the input and output design of the system.
\end{abstract}

\section{Introduction}

Tourism information management system is the management of tourism information resources in the system. Along with the increase in tourist information on the type and quantity, and expansion of the scope of tourism information management difficulty is further increased. Along with the popularization of computer, people are willing and habit through the computer to access to information resources, people can in the shortest possible time to obtain information, suitable for their own travel plan is then developed, both economically and improve work efficiency, to achieve the twice the result with half the effort. In this paper, design and development of tourism information management system will provide a platform for operation, to classify the information management, and specific aspects of tourism information involved as the basis of module partition. The concrete content includes: system login, the administrator module, tourism information input module, query module, information modification module, and exit system module, etc., and in these modules completed to add, modify, delete, query and other functions.

\section{Demand analysis and design of the system}

Feasibility study tourism management system . The computer information system, can effectively help tourism enterprises to optimize. Make is in a dominant position in the industry, and relatively long-term to maintain this strategic advantage. The development of the feasibility study of this system is as follows:

1) Technical feasibility: technical feasibility analysis mainly analyzes the mechanical conditions can be completed smoothly development work, the hardware and software can meet the need. This system USES the JSP to develop friendly and beautiful interface, easy for users to figure out, operation. Using SQL SERVER 2000 database management systems, it's fast, small volume, maintain data integrity, security, at the same time. Therefore the development platform of this system has been mature and feasible. Hardware, in today's rapid development of science and technology, hardware updates faster and faster speed, reliability higher and higher, the price is cheaper and cheaper, so the hardware platform can satisfy the system need.

2) Economic feasibility: the estimate of the costs and the analytical cost/benefit calculation of now, the system can bring certain economic benefits, improve the efficiency of tourism information release. The system on the basis of convenient and efficient and quick, help managers to realize tourism information management, has certain application value management, have very good practical and development prospects.

3) Operational feasibility: determine whether software products eventually can be accepted by users, the user is able to operate the final product. This system does friendly interface as much as 
possible in the development of design and operation of human nature, make the operator to easily get started.

4) Conclusion: the system development goal is clear, in technology and economy, three aspects are feasible, and less in investment, rapid. So the system development is completely achievable.

The advantages of the system:

1) A certain number of tourist attractions related information are available for processing. Tourists understand attraction various related information conveniently, and can improve and improve the management level, the attractions themselves do a better propaganda and promotion.

2 ) System according to the actual situation of the information collection as the background, system including the basic situation of each scenic spot is introduced

3 ) Management after login system, various complicated management through the system to manage, with the least amount of manpower and material resources to improve the management level.

4) Managers deal with various affairs through the system. The front desk of the user operation convenience simple, highlight the characteristics of tourism and has a beautiful interface design, guarantees the system safe and stable operation.

5 ) Administrator for the change of information, to manage information, ensure that the latest information and accuracy, and easy to daily operation and maintenance.

System requirements analysis. Depending on the demand of the tourism information management, we are attractions, tourist common problems such as tourism related information management. Mainly including guide attractions information management, information management, tourist information management as well as the system administrator information management from several aspects such as content. These aspects include the entry and query of information, and the updated information.

According to the overall functional requirements, described the specific functional requirements are as follows:

5) Attractions information management of the functional requirements: When the user was connected, can enter the scenic area management information page, the scenic spot can be input, modify, delete, query, resort information including the resort's name, address, contact phone number. Depending on update or change, attractions information input, modify, and delete and query operations.

6) The tour guide information management function requirements: Tour in the travel industry has a very important role, so have a clear guide information for tourists, convenient for tourists and tour guide at the same time the information on the travel company is required, the guide member information management, including some of the most basic information, such as the tour guide name, age, gender, phone number, and guide in the scenic spot, now enter the page of the tour guide information management can be seen, at the same time, the administrator can edit the information to the tour guide, add, modify, query, etc., can also query the nearest attractions.

7) Tourist information management functional requirements: Because this system is a very simple system for tourist information, manage in order to facilitate the travel agency for tourists, in this system provides some basic information to visitors, including tourists such as name, gender, and contact information, can be added to the tourist information, modify, delete, and query.

8) The user information management function requirements: The user information management is information management for the administrator to add, modify, query, and delete, the main land use authorization for users on the system,

The concept of database structure design . Conceptual data model, also known as an information model and its connection with entities - E - R theory as the foundation, and expanded on this theory. It from the user's point of view of information modeling, it is used primarily for the concept of database design. Usually people concept into the real world the world first, and then the concept of the world into a machine. First the objective object in the real world for abstract Entity (Entity) and contact (Relationship), it does not depend on the specific computer system or a DBMS system, this is what we call the CDM model; Then converts the CDM to computer supported by a DBMS data model, this model is the physical data model, namely, product data management (PDM).

CDM is a set of a set of strict definition model elements, these elements of the model accurately describe the static characteristic and dynamic characteristic of the system and the integrity constraint condition, etc., including the data structure, data operation and integrity constraints. 
1)Data structure is expressed as entities and attributes;

2)Data manipulation are expressed as the entity of the records in the insert, delete, modify, query and other operations;

3)Data integrity constraints are expressed as its own integrity constraints (such as data types, examination, rules, etc.) and the data between the referential integrity constraints (such as contact, inheritance, etc.);

The logical structure of database design . Information is included in the design of system information, guide information, line information, administrator information, financial information, etc. This information can be abstracted as the system needed items of data and the data structure:

1)Attractions information table (attractions name, location, attractions contact phone number).

2)Guide information table (guide's name, gender, age, professional, guide's contact phone number, where the spots).

3)Tourist information table (visitor number, name, gender, age, contact phone number).

4)The administrator information table (user name and password).

Through the design of the above steps, you can find the corresponding database information table, such as table 1 for the attractions information table.

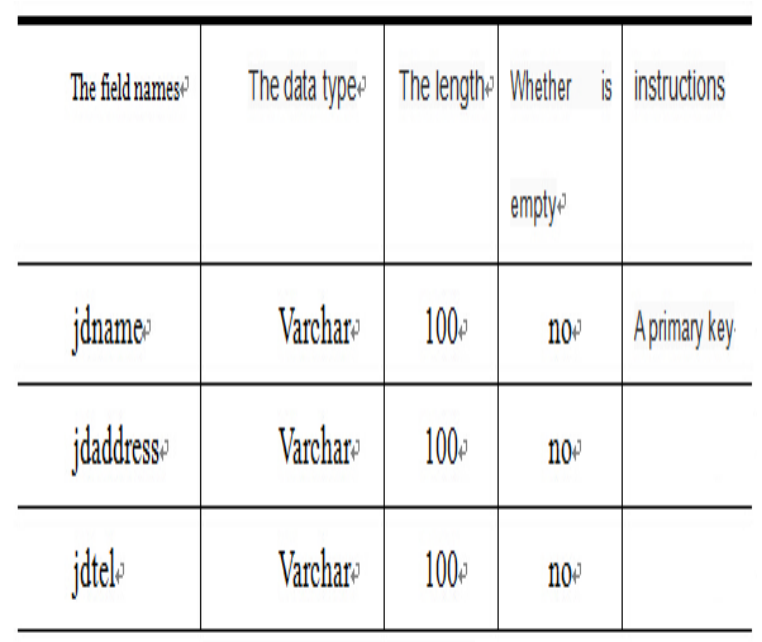

Figure 1. The information table

Database security and integrity. The integrity of the database refers to the correctness and consistency of the data. Database management system (DBMS) with a certain mechanism to check whether the data in the database is to satisfy the prescribed requirements - integrity constraint conditions, the constraint conditions that the data is a reflection of the semantic, as a part of the pattern in the database. The table and table set out in this system and the relation between each field in the table attribute constraints help promote help integrity.

Database security refers to the protection of databases in order to prevent the illegal use of data leakage, change, or destruction. Kept in a database system, a large number of data set, and for many users sharing directly, is a valuable information resource, system safety protection measures, it is more important, it can protect the database to prevent malicious damage and illegal access. In the system, the user main page by running into the system, must pass a logged in, only to browse this site attractions, view, query and other operations. User login user name is unique, through an encrypted password. In the user login, must enter the user name and password, and through the validation of database, at the same time to authenticate the user's permissions, this system to prevent illegal users and unauthorized users for illegal access and operation, ensure the security of this system. 


\section{The overall design of the system}

System function module design . According to the result of requirement analysis, according to the principle of "low coupling and high cohesion", the system will be divided into the following main function modules:

1) Tourism information management module: to achieve the main function including management, site management, guide information management, tourist information management, and administrators to manage their information.

2) Scenic spot management. The administrator can information in each scenic spot, including attractions name, location, features, etc.

3 ) The tour guide information management. The administrator can comprehensive information management of all the tour guide.

4 ) Tourist information management. The administrator to manage all visitors registration information.

9) The login module flow chart :The user to enter a user name and password, login screen after both right into the main interface functions, if it's not right requires entering a login and flow chart is as follows:

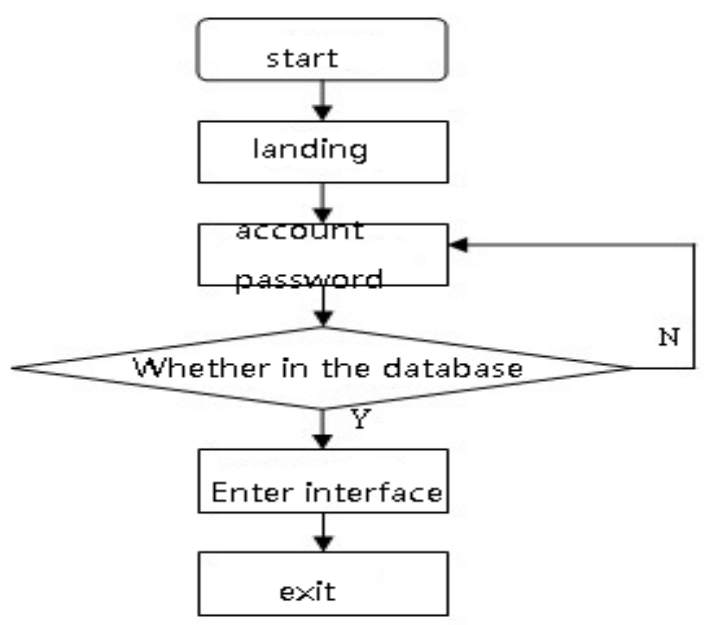

Figure 2. The flow chart of the user login

10) The administrator management flow chart :Administrators can be after a successful login, the relative information to add, delete, modify the etc operation, flow diagram as showed in the figure below:

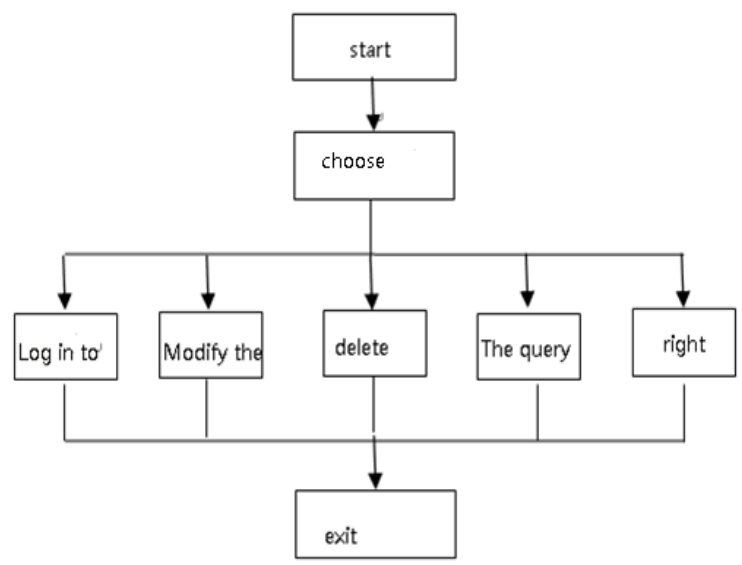

Figure 3. The administrator management flow chart 
Users enter the main interface (figure 4) can realize the management of tourism information, site management, guide information management, tourist information management, and administrators to manage their information. User management page code design in detail below:

$<$ html $>$

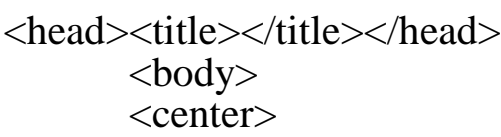

$<$ h1 $>$ Welcome to use tourism management system $</ \mathrm{h} 1>$

$<\mathrm{h} 2>$ $</ \mathrm{h} 2>$

$<$ h2> User management $</ \mathrm{h} 2>$

$<\mathrm{h} 2>$ $</ \mathrm{h} 2>$

$\langle$ h3 $\rangle\langle$ a href =yh_in.html $>$ entry $\langle/ \mathrm{a}\rangle\langle/ \mathrm{h} 3\rangle$

$\langle$ h3 $><$ a href =yh_up.html $>$ Modify the $\langle/$ a $\rangle\langle/$ h3 $>$

$\langle$ h3 $><$ a href $=$ yh_de.html $>$ delete $\langle/$ a $\rangle\langle/$ h3 $>$

$\langle$ h3 $><$ a href $=$ yh_se.html $>$ The query $\langle/$ a $\rangle\langle/$ h3 $\rangle$

$</$ center $>$

$\langle$ h3 $>$ permissions $\langle/$ h3 $>$

$</ h t m l>$

$</$ body $>$

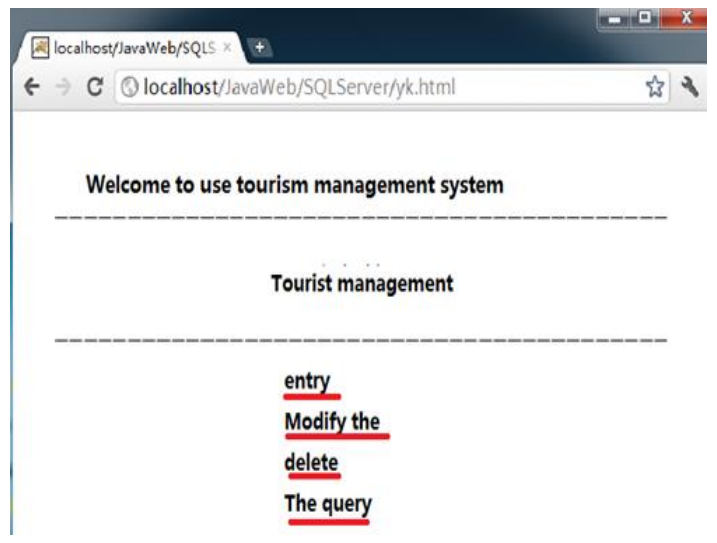

Figure 4. The user management main interface diagram

11) The query module flow chart :The user can enter the main interface to query operations, the input query condition, if found records are displayed, if found no record is returned to the input query conditions, the flow chart as showed in the figure below:

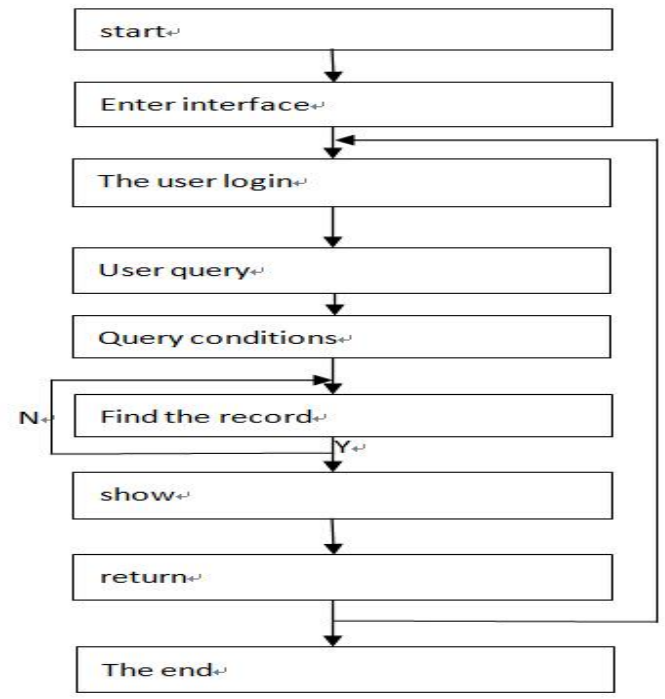

Figure 5. The flow chart of the query module 
The core module is convenient for the user on this site attractions related query, and returns the query results. User can choose according to their different needs corresponding keywords, the system according to the user's choice of keywords is related to query, get user query information. According to choose the type and input the keyword query, will query the attractions in the library and input fields exactly match attractions, the specific process as showed in figure 5 .

The system input and output design . The input interface of tourism management system mainly include user login, information query, information updates and tourists such as registration, enter the design contents include the following:

12) The content of the input data design

Include determining the input data item name. Data content, precision, numerical range. For example, in the user login, user input data include the username, password, cannot be free.

13) The correctness of input data validation

To ensure the correctness of the input data. When a user is connected, for example, must check the user name and password, only in the case of both the right to correct login.

\section{Conclusion}

The data design is a JSP as the programming language, mysql database based tourism management system, some basic information of the travel agency information management and processing of simple system, it can accurately and timely reflect the travel guide some basic information, contains the attractions and tourists, tourism management system is a powerful electronic data processing function, can greatly improve the work efficiency of management and operating personnel, at the same time, the system adopts the management model, combine modern decision means such as simulation, optimization, and provide effective information for the decision of the travel agency management.

\section{References}

[1] Haifan Zhang. An introduction to software engineering. Beijing: qinghua University press. 2008.

[2] Shixuan Sa, Shan Wang. Introduction to database system. Beijing: Higher education press. 2006.

[3] Bin Dai. Travel agency management. Beijing: tourism education press. 2005.

[4] Li Zhang. The SQL SEVER database principles and applications.

[5] ChengsanZhuang. "The principle of a database system and its application". Electronic industry press.

[6] Bole Shi, Baokang Ding. Book a course of a database system of higher education press, 2003, 2.

[7] Cai Changming, ding. Li Changjie. DreamweaverUltraDev4 introduction and improve. Tsinghua university press, 2001

[8] Xiong Songming, "standard of ASP.NET tutorial. Beijing aviation industry press, 2002

[9] Zhao Fengnian, the instance illustrated pages. Beijing mechanical industry publishing house, 2001

[10] XiBing, FIREWORKS 4 instances and operation. Beijing hope electronic publishing house, 2001 edition

[11] conan information, introduction to programming practice design. China railway publishing house, 2001

[12] Wang shan, Chen. The principle of database system tutorial. Tsinghua university press, 2000

[13] KuangKongWu, xiao-min wang. "information system analysis and design". Tsinghua university press, 2001 